\title{
Regional Variation of Pancreatic Cancer Incidence in the Nile Delta Region of Egypt over a Twelve-Year Period
}

\author{
Christina Baum (D), ${ }^{1}$ Amr S. Soliman ${ }^{D},{ }^{2}$ Heidi E. Brown ${ }^{1 D},{ }^{1}$ Ibrahim A. Seifeldin, ${ }^{3}$ \\ Mohamed Ramadan, ${ }^{3}$ Breanne Lott, ${ }^{4}$ An Nguyen, ${ }^{5}$ Ahmed El-Ghawalby, ${ }^{6}$ \\ and Ahmed Hablas $\left.{ }^{3}\right)^{3}$ \\ ${ }^{1}$ Department of Epidemiology \& Biostatistics, Mel and Enid Zuckerman College of Public Health, University of Arizona, Tucson, \\ AZ 85724, USA \\ ${ }^{2}$ Department of Community Health and Social Medicine, City University of New York Medical School, New York City, \\ NY 10031, USA \\ ${ }^{3}$ Gharbiah Cancer Society, Tanta, Gharbiah, Egypt \\ ${ }^{4}$ Department of Health Promotion Sciences, Mel and Enid Zuckerman College of Public Health, University of Arizona, Tucson, \\ AZ 85724, USA \\ ${ }^{5}$ Department of Global Health, Milken Institute School of Public Health, George Washington University, Washington, \\ DC 20052, USA \\ ${ }^{6}$ Department of Surgery, Liver Transplantation Unit, Gastrointestinal Surgery Center, College of Medicine, Mansoura University, \\ Mansoura, Egypt
}

Correspondence should be addressed to Amr S. Soliman; asoliman@med.cuny.edu

Received 1 March 2020; Revised 3 June 2020; Accepted 19 June 2020; Published 14 July 2020

Academic Editor: Eleanor Kane

Copyright $\odot 2020$ Christina Baum et al. This is an open access article distributed under the Creative Commons Attribution License, which permits unrestricted use, distribution, and reproduction in any medium, provided the original work is properly cited.

Background. Pancreatic cancer is one of the deadliest forms of cancer, with incidence rates rising in many countries around the world. Geographic variation in pancreatic cancer incidence has not been studied extensively, especially in low- and middle-income countries. The aim of this study was to characterize the distribution of pancreatic cancer incidence in the central Nile Delta region of Egypt and to examine differences by urban and rural patient residence using the nation's only population-based cancer registry. Methods. Utilizing the Gharbiah province population-based cancer registry, data were abstracted for 1,089 pancreatic cancer cases diagnosed over twelve years from 1999 to 2010. Age- and sex-specific incidence rates were calculated and compared for urban and rural areas of the eight districts of Gharbiah. Results. Age-adjusted incidence of pancreatic cancer within Gharbiah varied considerably by urban/rural patient residence and by district. Incidence rates were 1.3 times higher in urban compared to rural areas (4.45 per 100,000 in urban areas and 3.43 per 100,000 in rural areas). The highest incidence rates were observed in urban centers of Kotour, El Santa, and Kafr El-Zayat districts (12.94, 8.32, and 7.89, respectively). Conclusion. Incidence rates varied greatly by urban and rural areas and by district of residence in the Nile Delta region of Egypt. Future studies should examine potential environmental risk factors that may contribute to the geographic distribution of pancreatic cancer in this region.

\section{Introduction}

Pancreatic cancer is one of the deadliest forms of cancer, causing an estimated 432,000 deaths worldwide each year [1]. Its five-year survival rate of approximately $6 \%$ and its propensity for rapid progression make pancreatic cancer a significant global health issue [2]. Most cases are diagnosed in developed regions of the world, as defined by use of the Human Development Index and Gross Domestic Product, accounting for $55 \%$ of worldwide incidence [3]. However, both incidence and mortality rates are increasing in less developed countries [3]. Within Northern Africa, age- 
standardized incidence rates range from 1.0 per 100,000 in Sudan to 4.3 per 100,000 in Libya [4]. Little is known regarding the etiology of pancreatic cancer, but cigarette smoking, diabetes mellitus, exposure to occupational and environmental contamination, alcohol consumption, and genetics have been implicated as risk factors [5-11].

Due to the lethality of pancreatic cancer, mortality rates may act as surrogates for incidence rates. Mortality varies by province in Egypt, with northern districts having an average rate that is 2.85 times the rate of southern districts [12]. This variation may be due to differing environmental exposures, as the northern region of Egypt is also known to have some of the highest levels of soil and water pollution rates in the country [13-15]. A number of studies have identified exposure to environmental pollution, particularly heavy metals, pesticides, and hydrocarbons, as potential risk factors of pancreatic cancer [7, 16-19]. Our previous study included a comparison of the molecular pathology of pancreatic cancer tumors from patients residing in heavily polluted regions of the Nile Delta with the pathology of tumors from patients residing in low-pollution areas and found differences in the types and frequencies of tumor mutations, suggesting the influence of environmental factors [20]. Studying the geographic distribution of pancreatic cancer is essential to gaining a better understanding of how these risk factors may relate to pancreatic cancer etiology. Insights into the spatial distribution of cancer may also guide the geographic allocation of limited cancer prevention and control resources.

While mortality rates of pancreatic cancer across Egypt have been previously reported, this is the first study to describe the geographic distribution of incidence in Egypt. The purpose of this study is to characterize the distribution of pancreatic cancer incidence in the province of Gharbiah using population-based cancer registry data and to lay the groundwork for future etiologic studies to investigate the possible association between regional distribution of pancreatic cancer incidence and potential risk factors.

\section{Materials and Methods}

2.1. Study Population and Setting. The study population included all men and women 18 years of age and older who resided in the Gharbiah province and were diagnosed with pancreatic cancer between 1999 and 2010, as recorded in the Gharbiah Population-based Cancer Registry (GPCR) [21]. Data for this study were obtained electronically by abstracting clinical and demographic information of all eligible patients from the registry.

The province of Gharbiah is located in the central Nile Delta region and consists of eight districts with a total population of approximately 4 million people, according to the latest Egyptian census of 2006 [22]. Approximately $72 \%$ of the population reside in rural areas, with $28 \%$ residing in the eight capital cities of the eight districts [23]. Rural areas tend to be agricultural, while many urban areas in Gharbiah have undergone rapid urbanization and development [24].
2.2. Gharbiah Population-Based Cancer Registry (GPCR). The GPCR, located in the capital city of Tanta, was established in 1998 as a part of the Middle East Cancer Consortium (MECC) and received funding from the U.S. National Cancer Institute in Bethesda and the Egyptian Ministry of Health and Population until being discontinued due to lack of funding in 2011. The GPCR is known to be of high quality in terms of completeness and reliability. The International Association of Cancer Registries (IARC), the Surveillance Epidemiology and End Results (SEER) group of the National Cancer Institute, and the Department of Epidemiology of the University of California at Irvine have conducted validation and control quality checks with the MECC showing high data quality of this registry $[25,26]$. The percentage of death certificate only (DCO) cases is comparable between the GPCR (5.5\% in men and $4.7 \%$ in women), compared to the SEER registries $(2.5 \%$ and $3.4 \%$, respectively) [4]. The data from the GPCR have been included in two volumes of the international cancer publication, Cancer Incidence in Five Continents, and the GPCR is Egypt's only population-based cancer registry $[4,25]$.

The GPCR used active case finding to collect information on all cancer cases within the entire Gharbiah province. Pancreatic cancer cases included in the registry were classified based on the World Health Organization's ICD-O-2 coding for 1999-2000, followed by the ICD-O-3 coding from 2001 onward [27]. The following codes were included, representing all cases in the registry: C25.0, C25.1, C25.2, C25.3, C25.4, C25.7, C25.8, and C25.9. This includes all subtypes of pancreatic cancer. Information abstracted from the registry included case registry number, age at diagnosis, sex, district, urban/rural residence, basis of diagnosis, smoking status, occupation, and referral hospital. All personal identifiers were removed from the analyzed data to protect patient confidentiality. The study was approved by the University of Arizona's Institutional Review Board and the Gharbiah Cancer Society's Ethics Committee.

2.3. Census Data. Population data for each district of Gharbiah were obtained from the 1996 and 2006 censuses conducted by the Central Agency for Public Mobilization and Statistics (CAPMAS) [22]. The census consisted of 16 age categories of five-year age intervals for each district. It classified residence into two distinct categories; those who lived within the capital city of each district were considered urban, where the remaining towns and villages were considered rural. Each patient was assigned a residence code based on their residential address that aligned with CAMPAS coding to classify urban or rural cases. Using the total population of Gharbiah from the 1996 and 2006 census data, an annual growth rate (AGR) was calculated using the following formula: $A G R=((2006$ pop -1996 pop $) / 1996$ pop $) / 10$. This growth rate $(1.9 \%)$ was then used to estimate the yearly populations of each district, except for the district of Kotour. For Kotour, we observed a distinctly higher annual growth rate of $3.8 \%$, so we utilized this specific rate for Kotour only to ensure accurate population estimates. The population estimates formed the denominators in calculating age- and sexspecific incidence rates. 
2.4. Statistical Analysis. Data were obtained electronically from the GPCR, and descriptive statistics were generated using SAS Studio 3.8. Crude age- and sex-specific incidence rates (IR) were then calculated by dividing the number of pancreatic cancer cases per group (E) by the corresponding age- and sex-specific population estimates calculated with CAPMAS census data. Age-standardized rates were then calculated by direct standardization utilizing the Segi-Doll world standard [21]. Incidence rate ratios (IRRs) were also computed to compare urban and rural incidence rates of each district. The confidence intervals (CI) of the IRRs were calculated using the following formula: $\mathrm{CI}=\exp [\operatorname{In}(\mathrm{IR} 1 / \mathrm{IR} 2)] \pm$ $1.96 \times \sqrt{ }(1 / \mathrm{E} 1+1 / \mathrm{E} 2)$.

Shapefiles for Egyptian provinces and districts were downloaded from the Humanitarian Data Exchange and imported into ArcMap 10.6.1. A map layer created by the team was utilized to discriminate between urban and rural areas. Incidence data were imported into ArcMap in the form of a geodatabase, and choropleth maps were created.

\section{Results}

A total of 1,089 pancreatic cancer cases $(36.3 \%$ female and $63.7 \%$ male) were reported to the GPCR from 1999 to 2010 and included in this study (Table 1). Most of the study subjects resided in Tanta (26.2\%), Mehalla (26.8\%), and Kafr El-Zayat (10.8\%) districts, with $59.7 \%$ of cases residing in rural areas. The majority of the cases were diagnosed at the Tanta Cancer Center (50.5\%) and the Mansoura Gastroenterology Surgical Center (24.7\%). A total of 398 cases $(36.5 \%)$ were diagnosed using microscopic verification, including histology of either the primary tumor or metastasis, or cytology. Approximately half of the cases were younger than 60 years old (50.9\%). Information on smoking and occupation was not available for all patients (31.6\% missing values for smoking and $82.9 \%$ missing values for occupation) and therefore is excluded from Table 1.

Figure 1 and Table 2 present the age-adjusted incidence rates for urban and rural areas in all eight districts of Gharbiah, stratified by sex. Incidence rates varied by district, ranging from 2.46 per 100,000 in Zefta (southeastern Gharbiah) to 6.91 per 100,000 in Kotour (northwest Gharbiah). The districts with the highest overall incidence rates were Kotour, Kafr El-Zayat, and Mehalla (6.91, 4.18, and 3.93 per 100,000 , respectively). Figure 2 also displays the age-specific incidence rates, highlighting the correlation between pancreatic cancer and increasing age. As has been reported across the globe [2], our study shows that pancreatic cancer predominantly affects the older population, with $80.3 \%$ of cases diagnosed at age 50 years or above.

In all districts, incidence rates were higher in urban areas $(4.45 / 100,000)$ compared to rural areas $(3.43 / 100,000)$. The highest incidence rates were observed in urban centers of Kotour, El Santa, and Kafr El-Zayat districts (12.94, 8.32, and 7.89 per 100,000 , respectively). The districts with the highest urban-rural incidence rate ratios (IRRs) include El Santa $\quad(\mathrm{IRR}=2.48, \quad 95 \% \mathrm{CI}=2.18-2.81), \quad \mathrm{Kafr}$ El-Zayat $(\mathrm{IRR}=2.46,95 \% \mathrm{CI}=2.29-2.64)$, and Kotour $(\mathrm{IRR}=2.01$,
$95 \% \mathrm{CI}=1.57-2.41)$. The province-level (Gharbiah) agestandardized rate was 3.78 per 100,000 (4.87 and 2.72 per 100,000 for men and women, respectively) (Table 2).

\section{Discussion}

This was the first study to investigate the geographic distribution of pancreatic cancer incidence in Egypt. The study revealed two interesting trends. First, the results showed that incidence rates were significantly higher in urban compared to rural districts. Second, the incidence rates of pancreatic cancer varied greatly by district.

Regarding the difference between urban and rural areas, we found that urban areas had incidence rates that were 1.3 times the rates of rural areas (95\% CI: 1.29-1.31). The highest urban incidence was found in Kotour (14.28 per 100,000 in males and 11.27 per 100,000 in females) compared with the lowest rural incidence rate in Zefta (2.50 per 100,000 in males and 1.87 per 100,000 in females). These findings are in concordance with two studies conducted in China which demonstrated that pancreatic cancer incidence and mortality rates are higher in urban compared to rural areas [28, 29]. Another study which estimated pancreatic cancer incidence using hospitalization data in Brazil also found that estimated incidence rates were higher in urban compared to rural regions [30]. These findings were hypothesized to be related to the process of urbanization and the risk factors that accompany socioeconomic development such as increased smoking rates, adoption of westernized diets, lack of exercise, increased diabetes rates, changes in the urban environment, and improvement in diagnosis [28-30]. One study in Japan, however, found that there were no significant differences in pancreatic cancer mortality between metropolises, cities, and counties but offered no explanation for these results, which may have differed from other studies due to the manner in which municipalities were classified [31].

We believe it is unlikely that the observed differences between urban and rural rates were due to disparities in access to medical care. Across Egypt, 95\% of the population lives within $5 \mathrm{~km}$ of a primary health care facility [32]. Within Gharbiah, a large number of health care facilities in addition to prolific and inexpensive transportation options facilitate accessibility of medical services. The most distant location in Gharbiah is less than $50 \mathrm{~km}$ from either of the top two pancreatic cancer diagnosing clinics (Tanta Cancer Center and Mansoura Gastroenterology Center), with smaller health centers in the capital cities less than $20 \mathrm{~km}$ away from the most rural areas. The Ministry of Health and Population also provides free health care to all citizens, minimizing health care cost as a factor influencing accessibility [33]. These factors give us assurance that urban and rural differences are not related to accessibility and affordability of health care.

Differences in urban and rural lifestyles and occupational exposures are likely to contribute to the observed differences in urban and rural incidence rates. Obesity and diabetes have been shown to vary significantly between urban and rural parts of Egypt; according to a 1995 study, $49 \%$ of urban populations with higher incomes in Egypt were obese and 20\% 
TABLE 1: Clinical and demographic characteristics of 1,089 pancreatic cancer cases in Gharbiah during the period of 1999-2010 by urban and rural places of residence.

\begin{tabular}{|c|c|c|c|c|}
\hline Variable & Description & Urban no. (\%) & Rural no. (\%) & Total cases \\
\hline \multirow{13}{*}{ Year of diagnosis } & 1999 & $36(8.20)$ & $31(4.77)$ & $67(6.15)$ \\
\hline & 2000 & $30(6.83)$ & $38(5.85)$ & $68(6.24)$ \\
\hline & 2001 & $29(6.61)$ & $35(5.38)$ & $64(5.88)$ \\
\hline & 2002 & $41(9.34)$ & $42(6.46)$ & $83(7.62)$ \\
\hline & 2003 & $36(8.20)$ & $45(6.92)$ & $81(7.44)$ \\
\hline & 2004 & $44(10.02)$ & $44(6.77)$ & $88(8.08)$ \\
\hline & 2005 & $29(6.61)$ & $62(9.54)$ & $91(8.36)$ \\
\hline & 2006 & $42(9.57)$ & $58(8.92)$ & $100(9.18)$ \\
\hline & 2007 & $39(8.88)$ & $65(10.00)$ & $104(9.56)$ \\
\hline & 2008 & $31(7.06)$ & $77(11.85)$ & $108(9.92)$ \\
\hline & 2009 & $44(10.02)$ & $83(12.77)$ & $127(11.66)$ \\
\hline & 2010 & $38(8.66)$ & $70(10.77)$ & $108(9.92)$ \\
\hline & All years & 439 & 650 & 1089 \\
\hline \multirow{12}{*}{ Age } & $0-24$ & $1(0.23)$ & $3(0.46)$ & $4(0.37)$ \\
\hline & $25-29$ & $0(0.00)$ & $3(0.46)$ & $3(0.28)$ \\
\hline & $30-34$ & $7(1.59)$ & $8(1.23)$ & $15(1.38)$ \\
\hline & $35-39$ & $13(2.96)$ & $23(3.54)$ & $36(3.31)$ \\
\hline & $40-44$ & $17(3.87)$ & $38(5.85)$ & $55(5.05)$ \\
\hline & $45-49$ & $37(8.43)$ & $65(10.00)$ & $102(9.37)$ \\
\hline & $50-54$ & $73(16.63)$ & $90(13.85)$ & $163(14.97)$ \\
\hline & $55-59$ & $71(16.17)$ & $105(16.15)$ & $176(16.16)$ \\
\hline & $60-64$ & $76(17.31)$ & $103(15.85)$ & $179(16.44)$ \\
\hline & $65-69$ & $52(11.85)$ & $84(12.92)$ & $136(12.49)$ \\
\hline & $70-74$ & $46(10.48)$ & $66(10.15)$ & $112(10.28)$ \\
\hline & $75+$ & $46(10.48)$ & $62(9.54)$ & $108(9.92)$ \\
\hline \multirow{2}{*}{ Sex } & Female & $273(62.19)$ & $421(64.77)$ & $694(63.73)$ \\
\hline & Male & $166(37.81)$ & $229(35.23)$ & $395(36.27)$ \\
\hline \multirow{8}{*}{ Basis of diagnosis ${ }^{1}$} & Death certificate only & $54(12.41)$ & $19(2.92)$ & $73(6.73)$ \\
\hline & CT scan/US/MRI & $60(13.79)$ & $128(19.69)$ & $188(17.33)$ \\
\hline & Exploratory surgery/ERCP & $103(23.68)$ & $158(24.31)$ & $261(24.06)$ \\
\hline & Radiology with tumor marker & $60(13.79)$ & $105(16.15)$ & $165(15.21)$ \\
\hline & Cytology/hematology & $9(2.07)$ & $12(1.85)$ & $21(1.94)$ \\
\hline & Histology of metastases & $32(7.36)$ & $87(13.38)$ & $119(10.96)$ \\
\hline & Histology of primary & $116(26.7)$ & $141(21.69)$ & $257(23.69)$ \\
\hline & Autopsy with histology & $1(0.23)$ & $0(0.00)$ & $1(0.09)$ \\
\hline \multirow{8}{*}{ District } & Tanta & $148(33.71)$ & $137(21.08)$ & $285(26.17)$ \\
\hline & Mehalla & $153(34.85)$ & $139(21.38)$ & $292(26.81)$ \\
\hline & Kafr El-Zayat & $46(10.48)$ & $72(11.08)$ & $118(10.84)$ \\
\hline & Zefta & $24(5.47)$ & $55(8.46)$ & $79(7.25)$ \\
\hline & Samanoud & $21(4.78)$ & $51(7.85)$ & $72(6.61)$ \\
\hline & El Santa & $19(4.33)$ & $81(12.46)$ & $100(9.18)$ \\
\hline & Kotour & $13(2.96)$ & $65(10.00)$ & $78(7.16)$ \\
\hline & Basyoun & $15(3.42)$ & $50(7.69)$ & $65(5.97)$ \\
\hline
\end{tabular}

${ }^{1}$ Four cases $(0.37 \%)$ had missing data on the basis of diagnosis. 

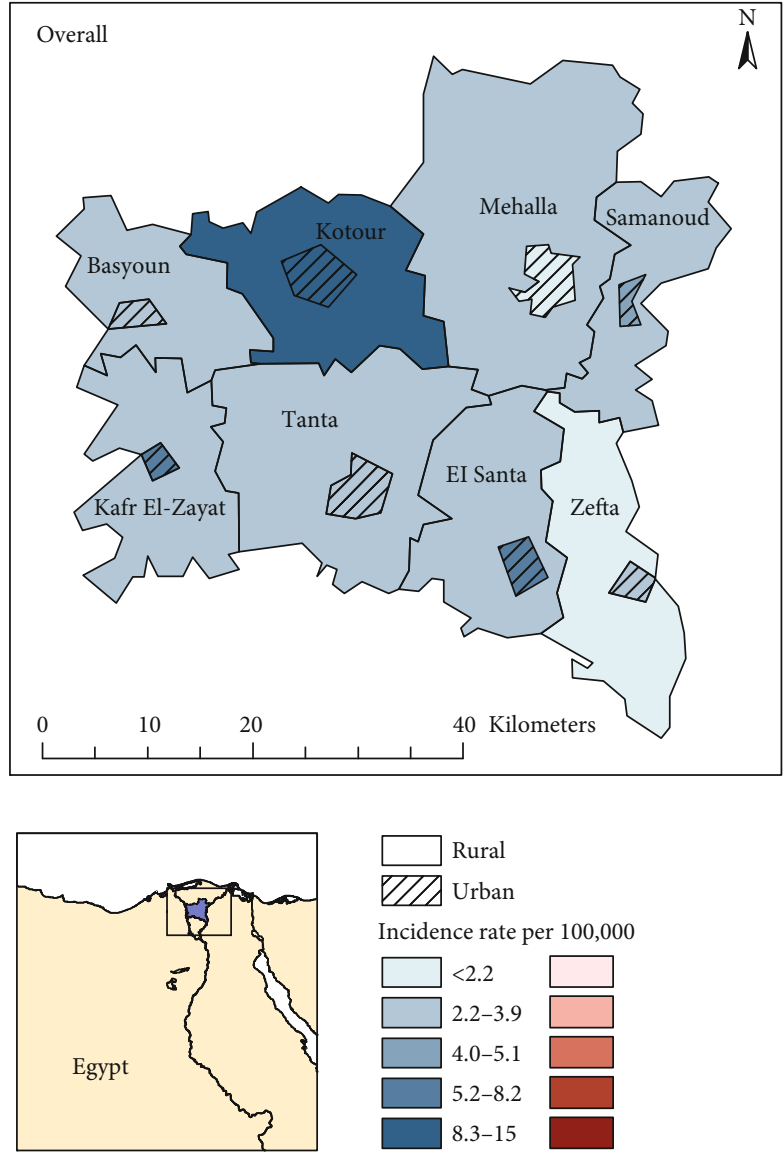

FIGURE 1: Map of pancreatic cancer incidence rates in urban and rural Gharbiah for males and females (1999-2010).
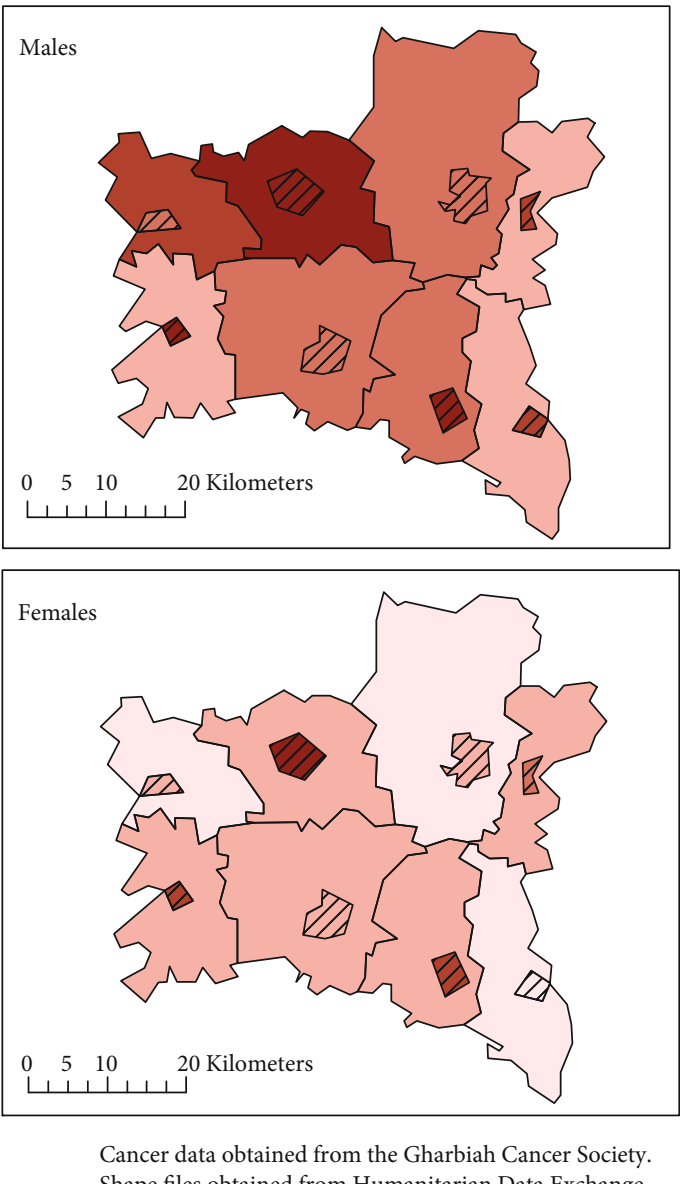

had diabetes, compared to rural populations where only $16 \%$ were obese and $4.9 \%$ had diabetes [34]. These figures have continued to climb since then, with an estimated $63.5 \%$ of Egyptian adults overweight or obese in 2016 and $15.6 \%$ overall diabetes prevalence in 2011 [35, 36]. A study in southern Egypt which examined dietary factors in relation to pancreatic cancer risk also showed that a higher caloric intake was associated with pancreatic cancer [37]. As urban residents have greater exposure to Westernized diets, characterized in part by energy-dense foods, dietary pattern differences between urban and rural households could be potentially contributing to elevated incidence for urban residents. While there may be differences in cigarette smoking habits between urban and rural populations, the prevalence of smoking in rural areas has not been well documented and lack of smoking status for cases in this study prevented analysis of any potential association [38]. In 2002, 35\% of Egyptian men and $1.6 \%$ of Egyptian women were estimated to be smokers, which may contribute to the differences in male and female incidence rates [39]. More recently, the World Health Organization estimated that $46.4 \%$ of men and $0.2 \%$ of women in Egypt were current cigarette smokers [40]. Other types of tobacco use such as waterpipes or "hookah" may also be important to investigate. One study found that rural Egyptian men perceived waterpipe use to be less harmful than cigarette smoking and were also less likely to contemplate quitting or avoiding tobacco use [41]. As smoking is one of the strongest risk factors for pancreatic cancer [2], further research would benefit from improved documentation of urban versus rural smoking and tobacco-use behavior.

In regard to occupational exposures, a hospital-based study conducted in the nearby province of Dakahleia found that farming, which is highly correlated with rural residence, was significantly associated with pancreatic cancer risk [42]. Our study, however, found that rural, predominantly agricultural areas, exhibited lower incidence rates. Another hospital-based study showed that pancreatic cancer cases who were both farmers and who reported a history of handling pesticides were 2.6 times more likely to have pancreatic cancer compared with farmers reporting no handling history [19]. It is possible, then, that urban occupational exposures are equally important as rural agricultural exposures or that the pattern of urban versus rural incidence rates which we observed was due to even more influential risk factors affecting urban populations. Other types of occupations occurring in urban areas with industrial exposure to pesticides and carcinogenic materials such as manufacturing, soldering, and metalworking have not been studied specifically in relation to pancreatic cancer in Egypt. 


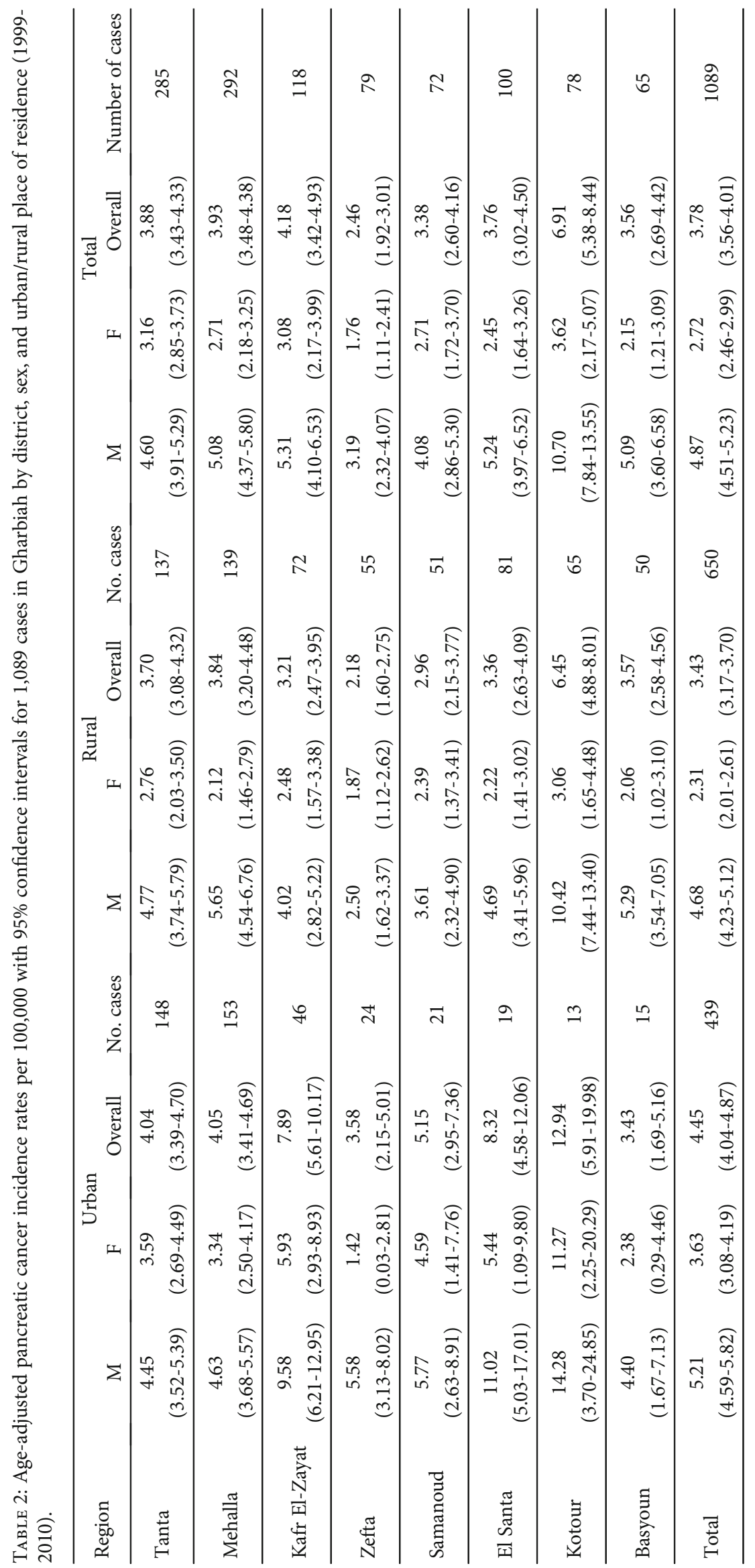




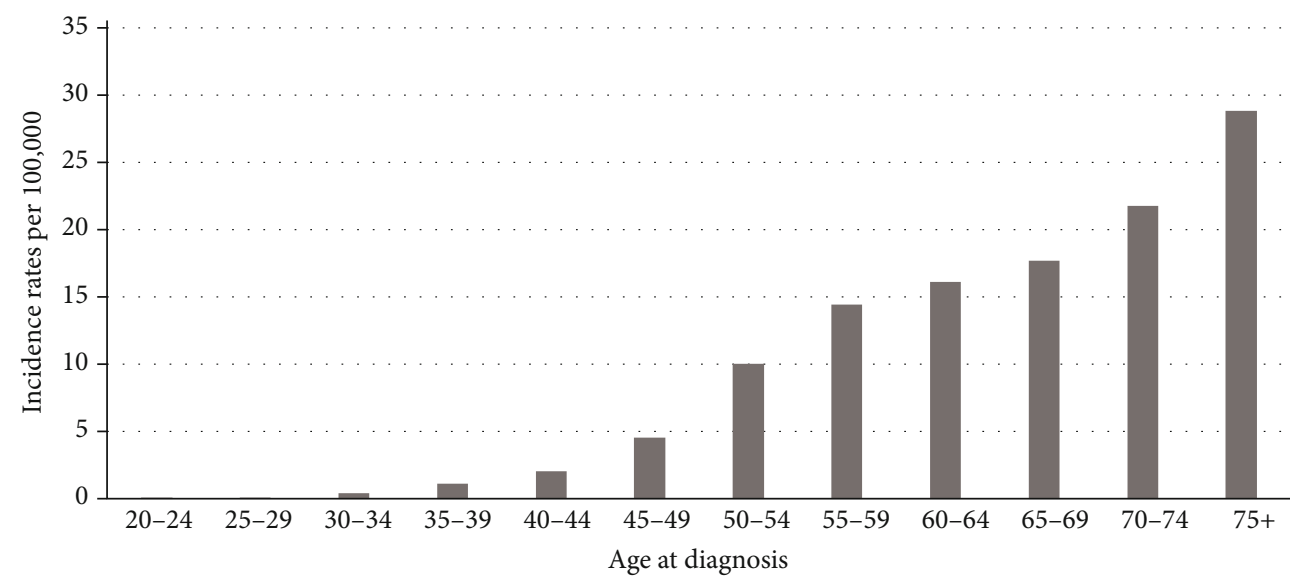

Figure 2: Age-specific pancreatic cancer incidence rates per 1,089 cases in the Gharbiah district of Egypt (1999-2010).

Regarding the observed regional variation, our results are consistent with other epidemiological studies demonstrating regional variation of pancreatic cancer incidence and mortality rates within the U.S., China, Japan, and France [12, 28, 43-45]. Our results are also in agreement with studies showing that pancreatic cancer incidence rates are lower in developing nations compared to northern, more developed countries $[3,12,46]$. The age-standardized rate of Gharbiah calculated in this study is 3.78 per 100,000 , compared to 7.7 per 100,000 in the United States [1].

As the districts in Gharbiah are relatively homogenous in terms of lifestyle, age distributions, and sex ratios, regional variation in incidence rates may be attributed to differences in exposure to environmental risk factors. It is well documented that the Nile Delta region has some of the highest levels of environmental contamination in Egypt by heavy metals, hydrocarbons, and pesticides; pollution levels are particularly high in urban and industrial areas [13, 47]. Cadmium, a known carcinogen and heavy metal, has also shown to be associated with pancreatic cancer cases and may contribute to the variation in incidence by district $[42,48]$.

This study has multiple strengths. Mainly, it is the first study to characterize the geographic distribution of pancreatic cancer incidence in Egypt. The GPCR is a high-quality registry that received regular quality checks by the IARC and is routinely cited by Cancer Incidence in Five Continents $[4,25]$. The study also utilized a large sample size for this rare cancer spanning twelve years. The population of Gharbiah is stable; people are likely to live in the same village or city for the entirety of their lives, and the migration rate throughout this period remained low [49]. Furthermore, 35\% of the cases used in this study were diagnosed by histopathology, a proportion comparable to other studies on pancreatic cancer from Western countries [50].

With these strengths, the study had limitations. There is the possibility that in Egypt and in other regions of the world, people may die of pancreatic cancer before seeking medical care or without obtaining a medical diagnosis first. However, we have no reason to believe that this would lead to differential misclassification. Because population-based cancer registries are not designed for specific research investigations, the variables in the registry were limited to clinical and descrip- tive epidemiologic information. Well-designed case-control studies that can assess individual risk factors such as smoking frequency, occupational exposures, and diet are needed. Additionally, although we had a reasonable sample size of 1,089 patients for this rare cancer, we were unable to conduct analyses on a finer spatial resolution. Utilizing more nuanced urbanization classifications, such as distinguishing semiurban areas, would also be useful to examine whether there is a linear correlation between pancreatic cancer incidence and the rate of urbanization. The population estimates used to calculate incidence differed slightly from the years that cases were reported but were utilized based on the availability of census data from 1996 and 2006. Lastly, while we are able to provide rationale for the differential rates by urbanization, data were not available to definitively establish causality.

\section{Conclusions}

This study demonstrates that pancreatic cancer incidence is higher in urban compared to rural areas in Gharbiah, Egypt. The study also reveals geographic variation in pancreatic cancer incidence by district of residence. It highlights the importance of cancer registration in Egypt and a need for future registration to provide relevant public health insights. This study provides a preliminary examination into pancreatic cancer distribution in Egypt; however, we recommend the completion of more advanced geospatial analyses with larger datasets to confirm these initial findings. In a country undergoing great urban growth, understanding the epidemiology of pancreatic cancer and investigating the risk factors which may vary between urban and rural settings and between districts will be essential for gaining a better understanding of the disease etiology and for guiding cancer prevention and control strategies.

\section{Data Availability}

All data used in the study were provided by the Gharbiah Cancer Society. Data are made available to researchers following a formal proposal for a specific study and request for data. 


\section{Conflicts of Interest}

The authors declare that there is no conflict of interest regarding the publication of this article.

\section{Acknowledgments}

We are grateful for Dr. Mohamed Hablas, Khaled Daboos, and other personnel at the Gharbiah Cancer Society for their assistance and support throughout this study. This work was supported by the Cancer Epidemiology Education in Special Populations (CEESP) program through a National Institute of Health/National Cancer Institute R25 grant [grant number CA112383].

\section{References}

[1] F. Bray, J. Ferlay, I. Soerjomataram, R. L. Siegel, L. A. Torre, and A. Jemal, "Global cancer statistics 2018: GLOBOCAN estimates of incidence and mortality worldwide for 36 cancers in 185 countries," CA: a Cancer Journal for Clinicians, vol. 68, no. 6, pp. 394-424, 2018.

[2] M. Ilic and I. Ilic, "Epidemiology of pancreatic cancer," World Journal of Gastroenterology, vol. 22, no. 44, p. 9694, 2016.

[3] M. C. S. Wong, J. Y. Jiang, M. Liang, Y. Fang, M. S. Yeung, and J. J. Y. Sung, "Global temporal patterns of pancreatic cancer and association with socioeconomic development," Scientific Reports, vol. 7, no. 1, pp. 3165-3169, 2017.

[4] D. Forman, F. Bray, D. Brewster et al., Cancer incidence in five continents, vol. X, IARC Scientific Publications, Lyon, France, 2014.

[5] S. O. Antwi, E. C. Eckert, C. V. Sabaque et al., "Exposure to environmental chemicals and heavy metals, and risk of pancreatic cancer," Cancer Causes \& Control, vol. 26, no. 11, pp. 1583-1591, 2015.

[6] J. P. Fryzek, D. H. Garabrant, S. D. Harlow et al., "A casecontrol study of self-reported exposures to pesticides and pancreas cancer in southeastern Michigan," International Journal of Cancer, vol. 72, no. 1, pp. 62-67, 1997.

[7] K. P. Cantor and W. Silberman, "Mortality among aerial pesticide applicators and flight instructors: follow-up from 19651988," American Journal of Industrial Medicine, vol. 36, no. 2, pp. 239-247, 1999.

[8] T. Y. Flanders and W. D. Foulkes, "Pancreatic adenocarcinoma: epidemiology and genetics," Journal of Medical Genetics, vol. 33, no. 11, pp. 889-898, 1996.

[9] M. Korc, C. Y. Jeon, M. Edderkaoui, S. J. Pandol, and M. S. Petrov, "Tobacco and alcohol as risk factors for pancreatic cancer," Best Practice \& Research. Clinical Gastroenterology, vol. 31, no. 5, pp. 529-536, 2017.

[10] C. Bosetti, E. Lucenteforte, D. T. Silverman et al., "Cigarette smoking and pancreatic cancer: an analysis from the International Pancreatic Cancer Case-Control Consortium (PANC4)," Annals of Oncology, vol. 23, no. 7, pp. 1880-1888, 2012.

[11] P. Maisonneuve and A. B. Lowenfels, "Epidemiology of pancreatic cancer: an update," Digestive Diseases, vol. 28, no. 4-5, pp. 645-656, 2010.

[12] A. S. Soliman, Q. Zhang, T. Saleh et al., "Pancreatic cancer mortality in Egypt: comparison to the United States pancreatic cancer mortality rates," Cancer Detection and Prevention, vol. 30, no. 5, pp. 473-479, 2006.

[13] M. I. Badawy, R. A. Wahaab, and H. F. Abou Waly, "Petroleum and chlorinated hydrocarbons in water from Lake Manzala and associated canals," Bulletin of Environmental Contamination and Toxicology, vol. 55, no. 2, pp. 87-100, 1995.

[14] F. R. Siegel, M. L. Slaboda, and D. J. Stanley, "Metal pollution loading, Manzalah lagoon, Nile Delta, Egypt: implications for aquaculture," Environmental Geology, vol. 23, no. 2, pp. 8998, 1994.

[15] A. M. Abdel-Satar, M. H. Ali, and M. E. Goher, "Indices of water quality and metal pollution of Nile River, Egypt," The Egyptian Journal of Aquatic Research, vol. 43, no. 1, pp. 2129, 2017.

[16] G. Andreotti and D. T. Silverman, "Occupational risk factors and pancreatic cancer: a review of recent findings," Molecular Carcinogenesis, vol. 51, no. 1, pp. 98-108, 2012.

[17] K. Jaga and D. Brosius, "Pesticide exposure: human cancers on the horizon," Reviews on Environmental Health, vol. 14, no. 1, pp. 39-50, 1999.

[18] M. Santibañez, J. Vioque, J. Alguacil et al., "Occupational exposures and risk of pancreatic cancer," Occupational and Environmental Medicine, vol. 25, no. 10, pp. 721-730, 2010.

[19] A.-C. Lo, A. S. Soliman, N. El-Ghawalby et al., "Lifestyle, occupational, and reproductive factors in relation to pancreatic cancer risk," Pancreas, vol. 35, no. 2, pp. 120-129, 2007.

[20] A. S. Soliman, A. C. Lo, M. Banerjee et al., "Differences in K-ras and p53 gene mutations among pancreatic adenocarcinomas associated with regional environmental pollution," Carcinogenesis, vol. 28, no. 8, pp. 1794-1799, 2007.

[21] F. Bray, M. Colombet, L. Mery et al., Cancer incidence in five continents, Vol. XI, International Agency for Research on Cancer Scientific Publications, International Agency for Research on Cancer, Lyon, 2017.

[22] The Central Agency for Public Mobilization \& Statistics (CAPMAS) Statistical Year Book, CAPMAS, Cairo, Egypt, 2008.

[23] Egypt national report: Addis Ababa declaration on population \& development in Africa beyond 2014, UNFPA Egypt Country Office, Ministry of Planning and Administrative Reform, 2018.

[24] M. K. Hassanein, "Impact of urbanization on agricultural land losses and climate change," Journal of American Science, vol. 10, no. 1, pp. 99-107, 2014.

[25] M. Curado, B. Edwards, H. Shin et al., Cancer incidence in five continents volume IX, International Agency for Research on Cancer Scientific Publications, Lyon, 2007.

[26] H. Anton-Culver, J. Chang, F. Bray et al., "Cancer burden in four countries of the Middle East Cancer Consortium (Cyprus; Jordan; Israel; Izmir (Turkey)) with comparison to the United States surveillance; epidemiology and end results program," Cancer Epidemiology, vol. 44, pp. 195202, 2016.

[27] A. Fritz, C. Percy, A. Jack et al., International Classification of Diseases of Oncology, Switzerland, Geneva, 3rd edition, 2000.

[28] X. Jia, P. Du, K. Wu et al., "Pancreatic cancer mortality in China," Pancreas, vol. 47, no. 2, pp. 233-237, 2018.

[29] W. Chen, R. Zheng, H. Zeng, S. Zhang, and J. He, “Annual report on status of cancer in China, 2011," Chinese Journal of Cancer Research, vol. 27, no. 1, pp. 2-12, 2015.

[30] L. M. Perrotta de Souza, J. P. L. Moreira, H. S. Fogaça, R. R. Luiz, and H. S. de Souza, "Pancreatic cancer incidence and 
lethality rates in Brazil," Pancreas, vol. 46, no. 5, pp. 699-706, 2017.

[31] I. Kato, K. Tajima, T. Kuroishi, and S. Tominaga, "Latitude and pancreatic cancer," Japanese Journal of Clinical Oncology, vol. 15, no. 2, pp. 403-413, 1985.

[32] World Health Organization regional office for the Eastern Mediterranean, “Egypt Health Profile 2015," 2015, http:// www.apho.org.uk/resource/view.aspx?RID=171709.

[33] H. Elgazzar, "Income and the use of health care: an empirical study of Egypt and Lebanon," Health Economics, Policy and Law, vol. 4, no. 4, pp. 445-478, 2009.

[34] W. H. Herman, M. A. Ali, R. E. Aubert et al., "Diabetes mellitus in Egypt: risk factors and prevalence," Diabetic Medicine, vol. 12, no. 12, pp. 1126-1131, 1995.

[35] R. Hegazi, M. El-Gamal, N. Abdel-Hady, and O. Hamdy, "Epidemiology of and risk factors for type 2 diabetes in Egypt," Annals of Global Health, vol. 81, no. 6, pp. 814$820,2018$.

[36] Global Health Observatory Data: Overweight and obesity, World Health Organization, 2016, https://www.who.int/gho/ ncd/risk_factors/overweight_obesity/obesity_adults/en/.

[37] M. G. Abdelrehim, E. M. Mahfouz, A. A. Ewis, A. E. Seedhom, H. M. Afifi, and F. M. Shebl, "Dietary factors associated with pancreatic cancer risk in Minia, Egypt: principal component analysis," Asian Pacific Journal of Cancer Prevention, vol. 19, no. 2, pp. 449-455, 2018.

[38] S. Fouda, M. Kelany, N. Moustafa et al., "Tobacco smoking in Egypt: a scoping literature review of its epidemiology and control measures," Eastern Mediterranean Health Journal, vol. 24, no. 2,2018 .

[39] J. Mackay, M. Eriksen, and M. P. Eriksen, "The Tobacco Atlas," in Myriad Editions Limited, P. Jerem and L. Candida, Eds., Geneva, Switzerland:, 2002.

[40] "WHO report on the global tobacco epidemic," 2017, https:// www.who.int/tobacco/surveillance/policy/country_profile/egy .pdf?ua $=1$.

[41] P. N. Singh, J. Neergaard, J. S. Job et al., "Differences in health and religious beliefs about tobacco use among waterpipe users in the rural male population of Egypt," Journal of Religion and Health, vol. 51, no. 4, pp. 1216-1225, 2012.

[42] A. M. Kriegel, A. S. Soliman, Q. Zhang et al., "Serum cadmium levels in pancreatic cancer patients from the East Nile Delta region of Egypt," Environmental Health Perspectives, vol. 114, no. 1, pp. 113-119, 2006.

[43] L. Brotherton, M. Welton, and S. W. Robb, "Racial disparities of pancreatic cancer in Georgia: a county-wide comparison of incidence and mortality across the state, 2000-2011," Cancer Medicine, vol. 5, no. 1, pp. 100-110, 2016.

[44] S. Kinoshita, Y. Wagatsuma, and M. Okada, "Geographical distribution for malignant neoplasm of the pancreas in relation to selected climatic factors in Japan," International Journal of Health Geographics, vol. 6, no. 1, article 34, 2007.

[45] G. C. Biol, "Les cancers digestifs en France," Cancer, vol. 2, pp. 174-180, 1997.

[46] M. M. Fidler, I. Soerjomataram, and F. Bray, "A global view on cancer incidence and national levels of the human development index," International Journal of Cancer, vol. 139, no. 11, pp. 2436-2446, 2016.

[47] M. S. Shokr, A. A. El Baroudy, M. A. Fullen et al., "Spatial distribution of heavy metals in the middle Nile Delta of Egypt,"
International Soil and Water Conservation Research, vol. 4, no. 4, pp. 293-303, 2016.

[48] B. G. Luckett, L. J. Su, J. C. Rood, and E. T. H. Fontham, "Cadmium exposure and pancreatic cancer in South Louisiana," Journal of Environmental and Public Health, vol. 2012, 11 pages, 2012.

[49] B. McCormick and J. Wahba, Migration and Mobility in the Egyptian Labour Market, Economic Research Forum, 2004.

[50] D. Daniel, D. Von Hoff, R. Hruban, and M. Porta, "Commentary," Pancreatic Cancer, 113-117, 2005. 